\title{
A HODOGRAPHIC APPROXIMATION METHOD FOR ANALYZING THE FLUID MOTION THROUGH NETWORK PROFILES
}

\author{
KOVACS, A[dalbert] \& KOVACS, L[aura]
}

\begin{abstract}
We present a general approach for the study of the compressible fluid's permanent and planary motion through a network of profile grids. Based on the used mathematical model, we propose an analytic method by linearly approximating the compressible fluid's characteristic curve and the special network's turbine profile. As a result, a calculus algorithm is given for analyzing the compressible fluid's motion through profile grids. Our approach is generic, generalizes the method of C.C. Lin and can be used for any approximation type of the hodographic method.

Keywords: hodographic variables, hodographic approximation method, hydrodynamic network, Leray transposition
\end{abstract}

\section{INTRODUCTION}

The theory of profile grids has an important application in the design and improvement of modern turbo machines turbines. The mathematical models used in the study of profile grids crucially depend on the structure and the physical characteristics of the fluid's motion through profile grids. State-of-the-art techniques rely on one of the following two methods:

a) the integral equation method or the hydrodynamic singularities method. This method was used in [4] to develop a calculus algorithm based on the theory of $\mathrm{p}$ analytic complex functions in conjunction with the boundary elements method;

b) the hodographic approximation method. As detailed in [3], well-known hodographic methods include the Tschiaplighin-Demtchenko method, the KármánTschien method, and the C. Iacob method. Depending on which hodographic method is used, the complexity of calculations vary, and an analytic solution is derived as a result. As proved in [6], the Kármán-Tschien method can be used for the study of the compressible fluid's motion through profile grids.

In this paper we address the latter approach and give an analytic method for analysing the compressible fluid's motion through profile grids (Section 4). Our approach extends the work of C. C. Lin [6], and it is generic. By generic we mean that it can be used with all three aforementioned hodographic methods (Section 3). The key ingredient of our work comes with the linear approximation of the compressible fluid's motion through profile grids. The present article refines the theoretical framework of [2], and gives a calculus algorithm for analysing the compressible fluid's motion.

\section{PRELIMINARIES}

Let $z(x, y)$ denote the complex plane of the compressible fluid's motion, and consider a specialized network of profile grids. Let $t$ denote the scaling parameter, $\lambda$ the installation angle, $l$ the profile length, and $t / l$ the network density. The fluid's motion through the specialized network is the result from a source $(Q$, $\left.\Gamma_{1}\right)$ placed at $-\infty$, pointing to another source $\left(Q, \Gamma_{2}\right)$ which is placed at $+\infty$. As stated in [3], the motion's hydrodynamic parameters are defined by the following equations:

$$
\begin{gathered}
\Gamma_{1}=V_{1} t \sin \left(\lambda+\alpha_{1}\right), \Gamma_{2}=V_{2} t \sin \left(\lambda+\alpha_{2}\right), \Gamma=\Gamma_{1}-\Gamma_{2}, \\
Q=\varsigma_{1} \cdot t \cdot V_{1} \cos \left(\lambda+\alpha_{1}\right)=\varsigma_{2} \cdot t \cdot V_{2} \cos \left(\lambda+\alpha_{2}\right),
\end{gathered}
$$

where $V_{1}, V_{2}, \varsigma_{1}, \varsigma_{2}$, represent the asymptotic velocity, respectively the fluid's density, at $-\infty$ and $+\infty$, while $\alpha_{1}$, $\alpha_{2}$ denote the angles between these velocities and the $0 x$ axis. Finally, the fluid's velocity at $-\infty$ is $\vec{V}_{1}=V_{1} \cdot e^{i \alpha_{1}}$, whereas at $+\infty$ it becomes $\vec{V}_{2}=V_{2} \cdot e^{i \alpha_{2}}$.

Throughout this paper, we consider a planary, permanent and isentropic fluid's motion. The fluid's pressure $p$ and density $\varsigma$ satisfy thus the following characteristic equation:

$$
\frac{\mathrm{p}}{\varsigma^{\gamma}}=\text { const } \quad \text { such that } \quad \gamma=\frac{c_{p}}{c_{v}}
$$

where $c_{p}$ and $c_{v}$ are special temperature values at isobar and izochor conditions. Using equation (2), the fluid's motion through the network is determined by the following equations.

- The continuity equation (known as Euler equation):

$$
\operatorname{div}(\varsigma \cdot \vec{V})=0 \text { or } \frac{\partial}{\partial x}(\varsigma \cdot u)+\frac{\partial}{\partial y}(\varsigma \cdot v)=0
$$

where $\vec{V}$ is the fluid's velocity, while $u=V_{x}, v=V_{y}$ denote the axial components of the velocity $\vec{V}$.

- The equation of the irotational motion's potential: 


$$
\operatorname{rot} \vec{V}=0 \quad \text { or } \quad \frac{\partial v}{\partial x}-\frac{\partial u}{\partial y}=0
$$

We note that equations (3) and (3') yield the following partial differential equation system:

$$
\frac{\partial \varphi}{\partial x}=\frac{\varsigma_{0}}{\varsigma} \cdot \frac{\partial \psi}{\partial y}, \frac{\partial \varphi}{\partial y}=-\frac{\varsigma_{0}}{\varsigma} \frac{\partial \psi}{\partial x}, u=\frac{\partial \varphi}{\partial x}, v=\frac{\partial \varphi}{\partial y},
$$

where $\varsigma_{0}$ represents $\varsigma$ in the null-velocity point, whereas $\varphi$ is the velocity potential and $\psi$ is the flow rate function of the fluid's complex velocity.

Observe that equation (4) yields a system of nonlinear partial differential equations, and thus cannot be written as a solution of a linear differential system. As analysing the resulting nonlinear differential equations becomes cumbersome, various linearising solutions have been proposed. We name here only one approach and refer to [3] for more details.

- S. A. Tschiaplighin proposed in [7] to rewrite the quasi-linear system (4) into a linear equation system, by using the independent hodographic variables $(V, \theta)$ given below:

$$
V \cdot e^{-i \theta}=u-i v \quad \text { with } \quad u=V \cos \theta, \quad v=V \sin \theta
$$

For doing so, the observation made by S. A. Tschiaplighin was that there exists an ideal fluid whose nonlinear characteristic equation (2) can be replaced with the following linear characteristic equation:

$$
\mathrm{p}=\mathrm{C} \frac{1}{\varsigma}+\mathrm{C}^{\prime}
$$

where the constants $C$ and $C^{\prime}$ are determined by the used hodographic approximation and reflect the real physical conditions of the fluid.

Following the observation of S. A. Tschiaplighin, the study of the compressible fluid can equivalently be replaced by the study of the ideal compressible fluid, where the motion of the ideal fluid is determined by the constants $C$ and $C$ '. Depending on the deployed hodographic approximation method, there are essentially three possibilities for choosing $C$ and $C^{\prime}$, defined geometrically as follows.

a) In the case of the Tschiaplighin-Demtchenko method, the line determined by (2') is tangential to the isentropic curve in point $P_{0}$ corresponding to the nullvelocity;

b) In the situation of the Kármán-Tschien method, the line determined by (2') is tangential to the isentropic curve in pont $P_{1}$ corresponding to the velocity at infinity;

c) When using the $C$. Iacob method, the line determined by (2') joins the points $P_{0}$ and $P_{l}$ on the isentropic curve.

The observation of S. A. Tschiaplighin and the existence of the Tschiaplighin-type ideal fluid simplifies thus the analysis of the compressible fluid's motion. In particular, S. A. Tschiaplighin made use of equations (4) and (5) in conjunction with the functional transposition $\sigma=\sigma_{1}+\int_{\mathrm{v}_{1}}^{\mathrm{v}} \frac{\varsigma}{\varsigma_{0} \mathrm{~V}} \mathrm{dV}$ of B. Demtchenko. As a result, $\mathrm{S}$.

A. Tschiaplighin showed in [7] that the ideal's fluid subsonic motion is characterised by the following system of equations:

$$
\frac{\partial \theta}{\partial \varphi}=\frac{\partial \sigma}{\partial \psi}, \quad \frac{\partial \theta}{\partial \psi}=-\frac{\partial \sigma}{\partial \varphi}
$$

Let us note that equation (6) defines the CauchyRiemann conditions. These conditions show that $\omega=\theta+i \sigma$ is an analytic function of $f=\varphi+i \psi$. We recall that the function $\omega=\theta+i \sigma$ is the Levi-Civita function of the incompressible fluid in the ideal plane $(\zeta)$. Moreover, in the ideal plane $(\zeta)$, the complex potential of the incompressible fluid satisfies $f=\varphi+i \psi$.

The results of S. A. Tschiaplighin thus imply that, by using the hodographic approximation method, one only needs to establish a one-to-one correspondence between the compressible fluid's motion in plane $(z)$ and the incompressible fluid's motion in plane $(\zeta)$. Such a correspondence ensures that a simplified analysis of the incompressible fluid's motion also yields a valid analysis of the compressible fluid's motion. In [1], C. Iacob addressed this problem and found the right correspondence between the planes $(\mathrm{z})$ and $(\zeta)$, as follows. Using the complex potential $\mathrm{f}(\zeta)$ of the incompressible fluid's motion around profile $\mathrm{L}_{\mathrm{i}}$, one determines the compressible fluid's motion around profile L. However, as the compressible fluid's motion through network profiles might result in a circular motion, C. Iacob also showed in [1] that the aforementioned transformation cannot be applied for the study of circular motions.

\section{PRACTICAL USE OF THE HODOGRAPH APPROXIMATION METHOD}

Following the results S. A. Tschiaplighin and C. Iacob, analysing the compressible fluid's (circular and non-circular) motion only requires to effectively compute a one-to-one correspondence between the planes $(z)$ and $(\zeta)$. To this end, we use the J. Leray transposition of [5], as given below:

$$
\overline{\mathrm{w}}(\zeta)=\frac{1}{\mathrm{~h}(\zeta)} \frac{\mathrm{df}}{\mathrm{d} \zeta}=\frac{1}{\mathrm{~h}(\zeta)} \overline{\mathrm{V}_{\mathrm{i}}}
$$

where $h(\varsigma)$ is defined so that the correspoding network profiles are closed.

As given in [2], we thus obatin the following result.

Theorem [2]. In the hodographic approximation method, the correspondence between the incompressible fluid's circular motion from the plane $(\zeta)$ and the compressible fluid's circular motion from the plane $(z)$ is given by the following equations: 


$$
\begin{aligned}
& z=C_{1} \int h(\zeta) d \zeta+C_{2} \int \overline{\left(\frac{d f}{d \zeta}\right)^{2} \frac{d \zeta}{h(\zeta)}}, \\
& \frac{1}{V}=\frac{C_{1}}{V_{i}}|h(\zeta)|+C_{2} \frac{V_{i}}{\mid h(\zeta)}, \\
& \theta=\theta_{\mathrm{i}}+\arg \mathrm{h}(\zeta),
\end{aligned}
$$

where $V_{i}$ is the magnitude of the incompressible fluid's velocity, and $\theta_{i}$ is the hodographic variable corresponding to $\mathrm{V}_{\mathrm{i}}$. The constants $C_{1}$ and $C_{2}$ in equations (8)-(9) are determined by the used hodographic method, as follows. In the sequel, let $\mathrm{V}_{\infty}=\mathrm{V}_{1}$ denote the asymptotic velocity at $-\infty$ infinity, $c_{0}$ the sound velocity in the null-velocity point, and $\mathrm{c}_{\infty}$ the sound velocity at infinity.

- In the case of the Tschiaplighin - Demtchenko method, we have:

$$
\mathrm{C}_{1}=\frac{1}{2}\left(1+\sqrt{1+\mathrm{M}_{0}^{2}}\right), \quad \mathrm{C}_{2}=-\frac{1}{2 \mathrm{~V}_{\infty}^{2}}\left(1-\sqrt{1+\mathrm{M}_{0}^{2}}\right),
$$

where $\mathrm{M}_{0}=\frac{\mathrm{V}_{\infty}}{\mathrm{c}_{0}}$ is the so-called Tschiaplighin-number,

- For the Kármán-Tschien method, we have:

$$
\mathrm{C}_{1}=\frac{1}{2}\left(1+\frac{1}{\sqrt{1-\mathrm{M}_{\infty}^{2}}}\right), \mathrm{C}_{2}=-\frac{1}{2 \mathrm{~V}_{\infty}}\left(1-\frac{1}{\sqrt{1-\mathrm{M}_{\infty}^{2}}}\right),
$$

where $\mathrm{M}_{\infty}=\frac{\mathrm{V}_{\infty}}{\mathrm{c}_{\infty}}$ is the so-called Mach-number;

- In the case of the C. Iacob method, we have:

$$
\begin{gathered}
C_{1}=\frac{1}{2}\left[1+\left(1-\frac{\gamma-1}{2} M_{0}^{2}\right)^{-\frac{1}{\gamma}-1}\right] \\
C_{2}=\frac{\gamma}{2 c_{0}^{2}}\left[1-\left(1-\frac{\gamma-1}{2} M_{0}^{2}\right)^{\frac{\gamma}{\gamma-1}}\left[1+\left(1+\frac{\gamma-1}{2} M_{0}^{2}\right)^{-\frac{1}{\gamma-1}}\right]\right.
\end{gathered}
$$

Using the results of S. A. Tschiaplighin in conjuction with the above equations, a correspondence between the incompressible fluid's circular motion in $(\zeta)$ and the compressible fluid's circular motion in (z) is thus derived.

Let us make the following observation. In [6], C. C. Lin proved that the compressible fluid's circular motion can efficiently be analysed using the hodographic approximation method. The work of C. C. Lin is however only applicable to the Kármán-Tschien method, and cannot be used for arbitrary hodographic approximation methods. It this paper we address this limitation and generalise the results of $C . C$. Lin. With such a generalisation at hand, our approach can be used with any hodographic method, by appropriately choosing the constant values $C_{1}$ and $C_{2}$. In the rest of this section, we detail the main steps of our approach.

Note that the fluid's motion is periodic through the network profiles. It is therefore sufficient to only analyse the motion in the principal periodical strip $\mathrm{D}_{0}^{-}(\mathrm{z})$ of the base profile. We derive the following result.

Theorem. In the hodographic approximation method, the one-to-one correspondence between the incompressible fluid's circular motion in the principal periodical strip $\mathrm{D}_{0}^{-}(\zeta)$ and the compressible fluid's circular motion in $\mathrm{D}_{0}^{-}(\mathrm{z})$ is given by equations (8) (10).

Further, we compute the function $\mathrm{h}(\zeta)$ by considering the work of J. Leray given in [5], as follows. In the canonical region of $(\zeta)$, the incompressible fluid's motion domain will be chosen around the $|\zeta|=1$ unit radius circle. The complex potential of the incompressible fluid's motion around the $|\zeta|=1$ unit radius circle is thus determined by the following equation:

$$
\mathrm{f}(\zeta)=\frac{\mathrm{Q}-\mathrm{i} \Gamma_{1}}{2 \pi} \ln \frac{\zeta+\mathrm{R}}{\zeta-\mathrm{R}}+\frac{\mathrm{Q}+\mathrm{i} \Gamma_{1}}{2 \pi} \ln \frac{\zeta+\frac{1}{\mathrm{R}}}{\zeta-\frac{1}{\mathrm{R}}}-\mathrm{i} \frac{\Gamma}{2 \pi} \ln \frac{\zeta-\mathrm{R}}{\zeta-\frac{1}{\mathrm{R}}},
$$

where $Q, \Gamma, \Gamma_{1}$ are as given in equation (1). The value of $R$ in equation (12) is determined from the table given in [3] (cf. page 62 of [3]).

Using the computed value of $R$, the angle $\theta_{0}$ corresponding to the stagnation point is next computed from the relation given below:

$$
\operatorname{tg} \theta_{0}=\frac{R^{2}-1}{R^{2}+1} \operatorname{tg} \lambda
$$

As a result, we conclude the following theorem.

Theorem [5]. In the case of networks with straight discs, the J. Leray-type $h(\zeta)$ function has the following expression:

$$
h(\zeta)=\frac{\left(1-\frac{S}{\zeta}\right)\left(1-\frac{1}{\zeta} e^{i \theta_{0}}\right)^{1-\frac{\varepsilon}{\pi}}}{\left(1+\frac{1}{R \zeta}\right)\left(1-\frac{1}{R \zeta}\right)\left(1-\frac{T}{\zeta}\right)^{2-\frac{\varepsilon}{\pi}}},
$$


where $\varepsilon$ is the profile's boundary angle, $S$ denotes a point on the stagnation point's radius such that it is defined by $\theta_{0}$ at a $0.0125-0.15$ distance from the circle. We note that the position of point $\mathrm{T}$ in equation (14) is computed such that the network profiles corresponding to $h(\zeta)$ are closed. That is, point $\mathrm{T}$ is computed by satisfying the following relation:

$$
\tau=\omega_{2}-\omega_{1}=0
$$

where $\omega_{1}$ and $\omega_{2}$ are defined in [3] as follows:

$$
\begin{aligned}
& \omega_{1}=\frac{C_{1}+C_{2}\left(v_{1 i} e^{i \alpha_{1}}\right)^{2}}{C_{1}^{2}+C_{2}^{2} v_{1 i}^{4}} \cdot \omega, \\
& \omega_{2}=\frac{C_{1}+C_{2}\left(v_{2 i} e^{i \alpha_{2}}\right)^{2}}{C_{1}^{2}+C_{2}^{2} v_{2 i}^{4}} \cdot \omega
\end{aligned}
$$

In equation (16), $\omega$ denotes the periodicity of the network profiles.

From equations (15) and (16), the position of point $\mathrm{T}$ in equation (14) is finally inferred, by using the equation given below:

$$
C_{1}+C_{2}\left(V_{1 i} e^{i \alpha_{1}}\right)^{2}=h(-R)\left[C_{1}^{2}+C_{2}^{2} V_{1 i}^{4}\right]
$$

In equation (17), the velocities $\mathrm{V}_{1 \mathrm{i}}$ in are computed using the compressible fluid's asymptotic velocity $\mathrm{V}_{1}$ at infinity, as follows:

$$
\frac{1}{\mathrm{~V}_{1}}=\frac{\mathrm{C}_{1}}{\mathrm{~V}_{1 \mathrm{i}}}+\mathrm{C}_{2} \mathrm{~V}_{1 \mathrm{i}}
$$

As a result of these steps, the value of $h(\zeta)$ in (14) is hence obtained, and the J. Leray functional transposition is derived from equation (7). Using the computed Leray transposition, we thus established a one-to-one correspondence between the incompressible fluid's circular motion around the $|\zeta|=1$ unit circle and the compressible fluid's circular motion in plane (z).

\section{A CALCULUS ALGORITHM FOR DETER- MINING THE FLUID'S COMPLEX VELO- CITY}

We now have all ingredients for analysing the compressible fluid's motion. For doing so, we state and use the following algorithm.

1. We are given the entering and the exiting parameters from the network: V1, V2, $\alpha 1, \alpha 2, \varsigma 1, \varsigma 2$. Similarly, the parameters $\varsigma 0, \mathrm{p} 0, \mathrm{c} 0$ of the null-velocity point are given, and the network paramters $\lambda, \mathrm{t}, \mathrm{t} / \mathrm{l}$ and the values $\varepsilon, \gamma$ are known.

2. The hydrodynamical parameters $Q, \Gamma 1, \Gamma$ are determined from equation (1).
3. Depending on the used approximation method, the constant values of $\mathrm{C} 1, \mathrm{C} 2$ are computed from equations (11)-(11'”).

4. Using $\lambda$ and $t / 1$, the parameter $1 / \mathrm{R}$ is chosen from its table of values (cf. page 62 of [3]).

5. Using equation (13), $\theta 0$ is derived as a function of $\lambda$ and $1 / \mathrm{R}$

6. The position of point $\mathrm{T}$ is then calculated from equation (17), where $\mathrm{V} 1 \mathrm{i}$ are derived from equation (18).

7. The unit radius circle $|\zeta|=1$ is divided conform a chosen division with $\mathrm{n}$ points, and the coordinates of the division points $\mathrm{Mi}$, with $\mathrm{i}=1, \ldots, \mathrm{n}$, are further computed.

8. Using equations (12)-(14), the function values $\mathrm{f}(\zeta \mathrm{i})$ and $\left|h\left(\zeta_{\mathrm{i}}\right)\right|$ are inferred.

9. From equation (9), the velocities V(Mi) are derived.

\section{CONCLUSION}

We present a generalisation of the C. C. Lin method and give a calculus algorithm for analysing the compressible fluid's motion through profile grids. Our approach can be used with any variant of the hodographic approximation method, by appropriately choosing the constant values of $C_{1}$ and $C_{2}$. In particular, if the constants $C_{1}$ and $C_{2}$ are determined using the Kármán-Tschien approximation method, our algorithms yields the approach given by the C. C. Lin method. It is important to note that our algorithm solves both the direct and the inverse hydrodynamical problems of network profiles. Further work includes practical improvements of our method and experiments with our algorithm using different hodographic approximation methods.

\section{ACKNOWLEDGEMENTS}

The second author is supported by a FWF Hertha Firnberg Research grant (T425-N23).

\section{REFERENCES}

[1] Iacob, C. (1939). On the Slow Motion of the Ideal Compressible Fluid (in French), Ed. Portugalae Matematica, T. 1, pp. 209

[2] Kovacs, A. \& Kovacs, L. (2001). A Calculus Algorithm based on Hodographic Approximations, used in the Aerodynamics of Network Profiles (in German). Proceedings of the 9th Symposium of Mathematics and its Applications, Timisoara, Romania, ISSN 1224-6069, Boja, N. et al (Ed.), pp. 274-279, Ed. Politehnica, Timisoara, Romania

[3] Kovacs, A. (2005). Classical and Modern Mathematical Methods in the Hydrodynamics of Profile Grids (in German), Ed. Politehnica, ISBN 973-625-230-2, Timisoara, Romania, pp. 144

[4] Kovacs, A. \& Kovacs, L. (2011). Analyzing the Fluid Motion Through Network Profiles Using the Boundary Element Method. Annals and Proceedings of DAAAM 2011, Vienna, Austria, ISBN 978-3-901509-83-4, Katalinic, B. (Ed.), pp. 1147-1148, DAAAM International, Vienna, Austria

[5] Leray, J. (1946). The Mechanics of Compressible Fluids (in French). Centre d'Etude Sup. de Mécanique des Fluides, Paris, France, pp. 87-94

[6] Lin, C. C. (1949). On the Slow Motion of the Compressible Fluid through Airfoils (in French). Journal of Mathematics and Physics, Vol. XXVIII, No. 2, pp. 117

[7] Tschaplighin, S. A. (1914). The Theory of Network Profiles (in Russian). Ed. Mat.zbornik, T. 29, pp. 414 